Preventing the Sale of Fraudulent Gemstones using Non-Destructive X-Ray Fluoresence Spectroscopy

\author{
Mary S. Goldman, Dan L. Davis, Robert H. Clifford \\ Shimadzu Scientific Instruments Inc., Columbia, MD \\ msgoldman@shimadzu.com
}

As the old adage goes, beauty is in the eye of the beholder. Ultimately, in the world of jewelry, the unwitting buyer uses his or her naked eye to decide which baubles are the most beautiful and worthy of wear. The expert jeweler and gemologist, however, know that each gem is subjected to rigorous scientific examinations and standards to determine its value and authenticity.

As the number of methods for altering gems grows with technology, methods to counteract counterfeiters, who can take a worthless stone and alter it to make it appear like a more expensive gem, are essential to gem buyers and traders. They must be able to detect artificial stones.

Gemologists assess gemstones based on several standards such as chemical composition and purity. Chemical composition plays a significant role in evaluating gems, as two of the same gemstone can differ chemically. One of the most well known methods of nondestructive testing for detecting gem quality is X-ray fluorescence (XRF) spectroscopy. Increasingly in demand, XRF spectroscopy is an ideal technique for fighting the battle against fraud.

\section{Why X-ray?}

$\mathrm{X}$-ray is a commonly used technique because it is non-destructive and saves gemologists time and cost in product damage for testing. There are four X-ray techniques: X-ray diffraction (XRD), wavelength dispersive $\mathrm{X}$-ray spectroscopy (WDX), energy dispersive $\mathrm{X}$-ray spectroscopy (EDX), and X-ray fluorescence spectroscopy.

$\mathrm{XRD}$ is based on observing the scattering pattern of X-rays diffracted from a crystalline sample's atomic planes in order to determine its structure, composition, and other physical properties. XRD measurements provide routine identification and quantification of crystal phases in powder and polycrystalline samples. The result is referred to as the $\mathrm{X}$-ray diffraction pattern of the sample. Computer analysis of the peak position and intensities associated with this pattern enables qualitative analysis, lattice constant determination and stress determination of the sample. XRD is not suitable for the type of analysis discussed here, as the crystal structure of the specimens are not the issue.

The WDX spectroscopy method counts the number of X-rays of a specific wavelength diffracted by a crystal. X-rays focused on a sample fixed on the axis of the spectrometer are diffracted by the sample. The changes in the diffracted X-ray intensities are measured, recorded, and plotted against the rotation angles of the sample.

WDX only reads/counts the X-rays of a single wavelength, instead of analyzing a sample via a wide-range spectrum in one quantitative run, as with XRF. At times, WDX can produce better energy resolution and less background noise. However, such disadvantages as more analysis time, more sample damage and high cost outweigh the advantages for most gemologists.

EDX analysis produces results similar to XRF, i.e. a list of elements present in the specimen. To stimulate the emission of characteristic X-rays from a specimen, a high-energy beam of charged particles such as electrons or protons, or a beam of X-rays, is focused into the sample being studied. The abbreviation "EDX" is commonly associated with the "electron beam induced specimen characteristic
X-rays method," whereas "XRF" is associated with the "high-energy $\mathrm{X}$-ray induced specimen characteristic X-rays method." Accordingly, EDX is usually performed in a vacuum in an electron microscope, making it much more expensive, and often requires destructive specimen preparation.

How does XRF spectroscopy differ from these other methods? Technically, XRF is a form of EDX where a specimen's characteristic $\mathrm{X}$-rays are stimulated by high energy $\mathrm{X}$-rays and the technique can be performed in air and may often be performed with inexpensive bench-top or hand-held instruments. Fluorescent and scattered $\mathrm{x}$-rays are emitted from the specimen and detected using a highenergy resolution solid-state detector. A rapid analysis device, XRF spectroscopy easily differentiates quality gemstones from stones that are counterfeit or have undergone chemical enhancement by listing the elements present in the specimen and making it possible to determine the concentrations of each of the specimen's elements. In addition, unlike other test methods that may require the chipping or laser-cutting of a stone, XRF is completely nondestructive. It does not damage the stone in any way. XRF analysis can be used at any stage of the gem buying process, from raw materials to finished jewelry. By using qualitative and quantitative functions that identify any element from sodium to uranium, XRF analysis leaves little room for deception. XRF is, by far, the easiest of the techniques and the most cost-effective for screening consumer products.

There are two different techniques that can be used to perform an analysis. For unknown samples or samples where the user would like to know "what is in there," XRF can do a qualitative scan. This scan will produce a list of all elemental components found in the sample with atomic numbers greater than that of sodium. The second quantitative technique determines each element's concentration in

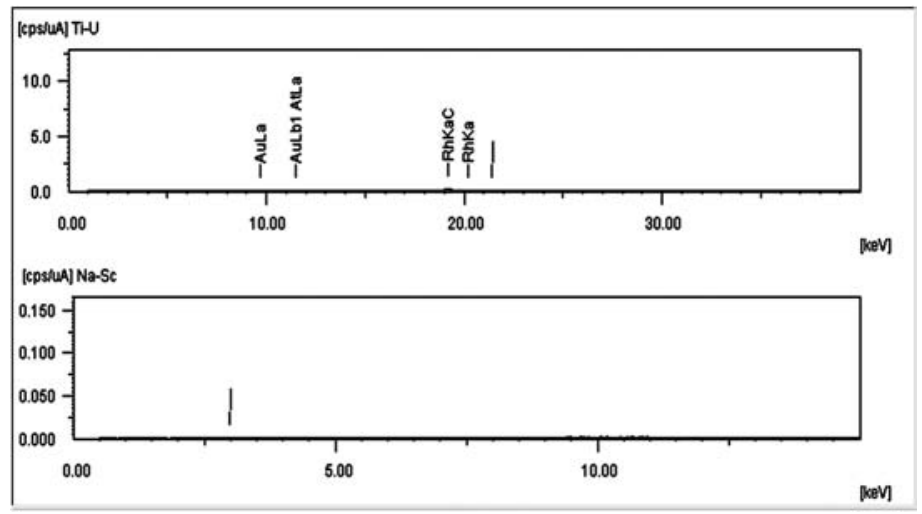

Analysis of a true diamond will result in no elements detected, as carbon's atomic number is lower than that of sodium [Figure 1]
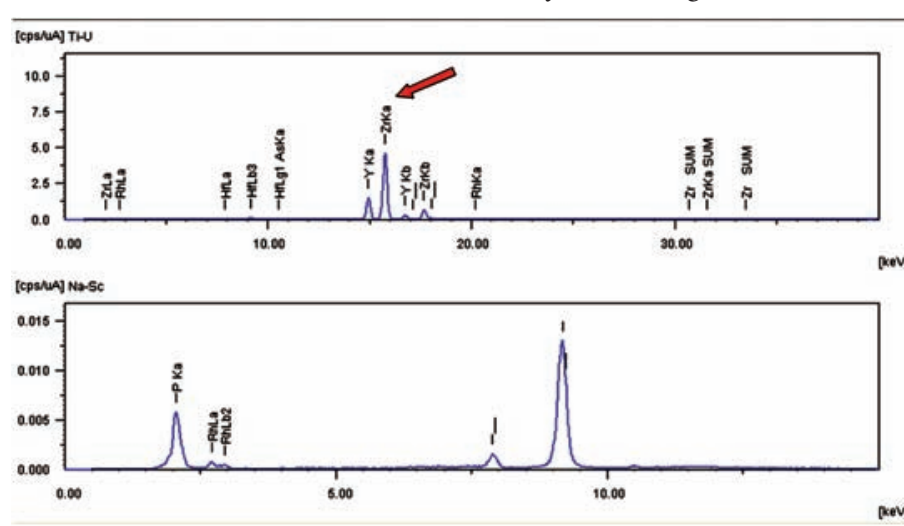

The analysis of a cubic zirconium diamond, with a similar appearance to a true diamond, will show peaks of zirconium along with other elements used as filler in the gem [Figure 2] 


\section{MIRA $\|_{\text {Schottky FE-SEM }}$}

\section{Value and Excellence in SEMs}

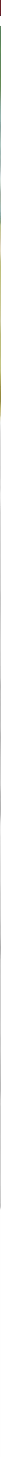

All as Tescan standard for the highest-quality imaging 
the specimen matrix. Once a gem's elemental and concentration parameters are determined, the results may be saved in the machine. All future samples of that particular gem and its testing method can be immediately selected at the push of a button for quick analysis.

An XRF spectrometer has no size requirement for testing materials, as its method corrects for undersized gem samples. The spectrometer can precisely measure gemstone areas utilizing analysis $\mathrm{X}$-ray spots as large as $10 \mathrm{~mm}$ to X-ray beam spots as small as 0.3 $\mathrm{mm}$. Gems smaller than $0.3 \mathrm{~mm}$ can be examined although there will be a greater amount of X-ray back scatter noise in the final data set.

\section{Crystal clear}

No gemstone crystal is absolutely pure. For example, any substance other than carbon found in a diamond is an impurity. Impurities can be in the form of discreet inclusions, or the foreign element may exist in solid solution within the gem's crystal lattice. While foreign elements can be unwanted, they also can be introduced on purpose to control a gem's properties such as clarity and color.

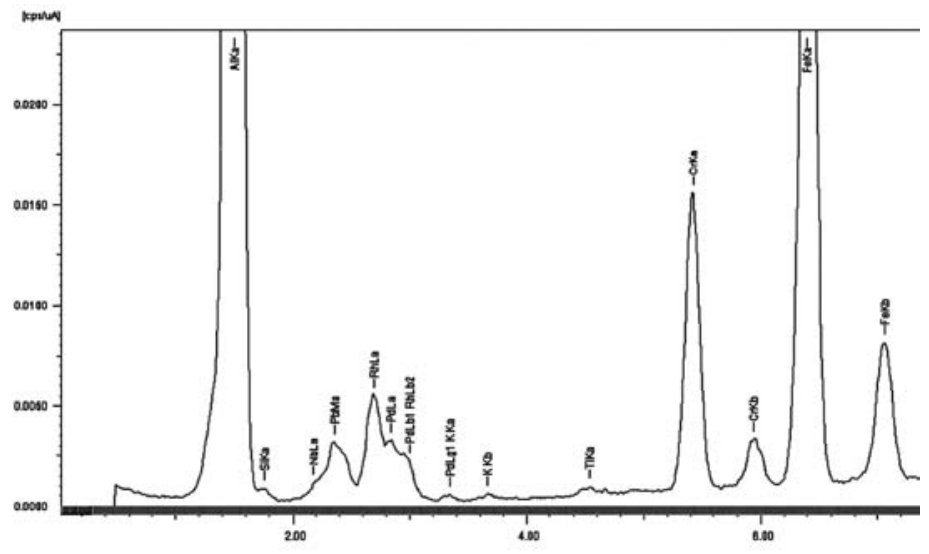

Figure 3 shows the data profile of a ruby.

When testing a gem to see if it is a true diamond, an XRF spectrometer can be used with a Rh-target X-ray tube as the source of the analyzing high-energy $\mathrm{X}$-rays. Analysis of a true diamond will result in no elements detected, as carbon's atomic number is lower than that of sodium [Figure 1]. The analysis of a cubic zirconium diamond, with a similar appearance to a true diamond, will show peaks of zirconium along with other elements used as filler in the gem [Figure 2]. When comparing the two spectra, it is easy for a gemologist to see which stone is authentic and which stone is not. The gemologist can also identify if any impurities were added to enhance the perceived quality of the stones.

Figure 3 shows the data profile of a ruby. The elemental structure of a ruby is $\mathrm{Al}_{2} \mathrm{O}_{3}: \mathrm{Cr}$, and natural stones may have rutile inclusions. Rutile has a titanium oxide structure $\left(\mathrm{TiO}_{2}\right)$, and can form needleshaped inclusions in the $\mathrm{Al}_{2} \mathrm{O}_{3}$ that causes an optical asterism that increases the gem's value. This profile shows aluminum, chromium, iron, and small amounts of titanium. If the stone were synthetic, the profile would indicate nickel, molybdenum, lanthanum, tungsten, platinum, lead, and bismuth. Because none of these elements appears, the gemologist is assured that the gem is authentic.

\section{Semi-precious stones}

To the untrained eye, some gemstones that appear the same physically may, in fact, be completely different stones. Take apatite and tourmaline, for example, which are virtually identical. Using XRF to analyze apatite and tourmaline reveals how two seemingly identical stones can be mistaken for one another. Following XRF analysis, the apatite stone spectrum [Figure 4] revealed a composition of calcium oxide and phosphorous pentoxide. These compounds
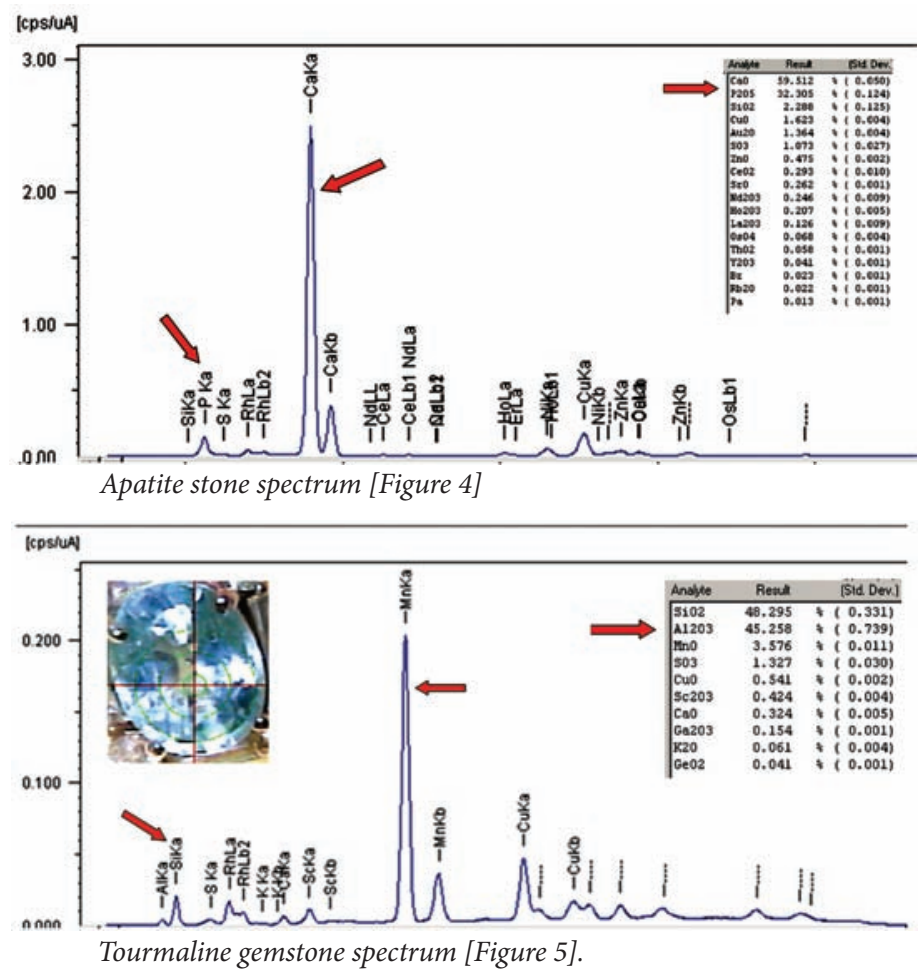

are not found naturally in the tourmaline group. Looking at the tourmaline gemstone spectrum [Figure 5] enables the gemologist to classify the tested stone into its specific mineral group. When the two spectra are compared, tourmaline - the more expensive gemstone - shows a manganese peak. Apatite, a less pricey stone, does not contain this element.

In another analysis using XRF, gemologists tested jade. Two types of jade, nephrite and jadeite, exist naturally. Nephrite is the rarer form and sells at a higher price. In each of these stones, a certain element is responsible for giving the jade its natural color.In the profile of green jadeite in Figure 6, chromium gives the stone its green color, and iron gives the jadeite a brownish hue. By looking at the data, gemologists can chemically infer that the stone is a natural green color. In this case, XRF was a quick and easy way to identify an artificially colored stone against naturally colored pieces.

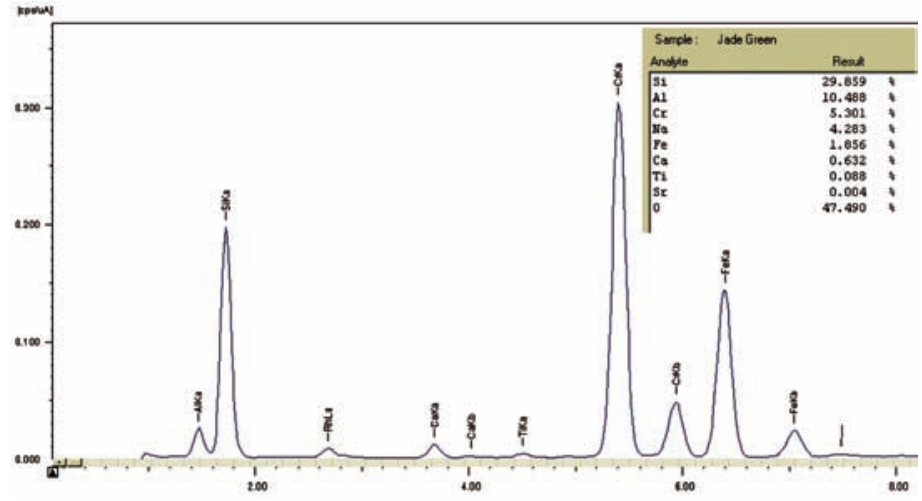

In the profile of green jadeite in Figure 6, chromium gives the stone its green color, and iron gives the jadeite a brownish hue.

\section{Conclusion}

Steps are being taken each day in laboratories to protect jewelers and buyers from jewel counterfeiting. XRF is a simple, rapid technique that can be easily integrated into the manufacturing process to rapidly screen gems before they are sold individually or appear as part of lavish baubles in the windows of your local jeweler. 
